
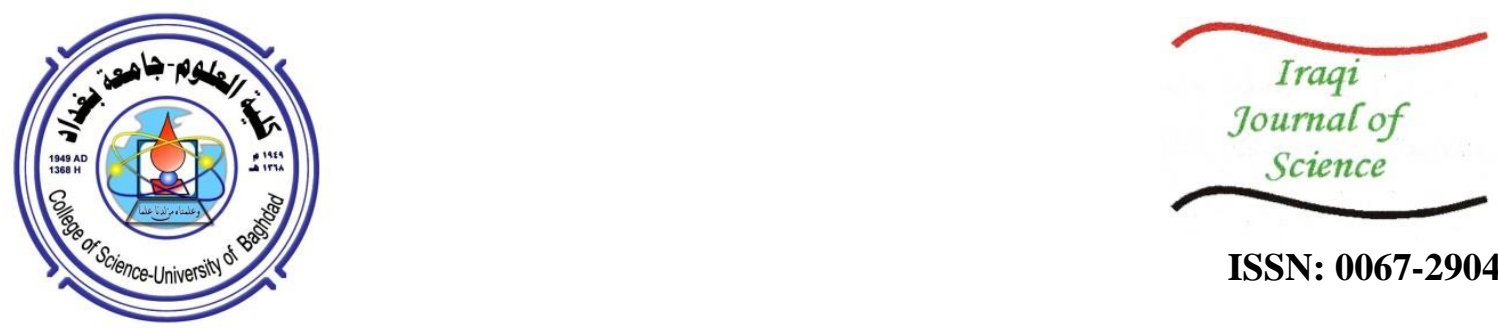

ISSN: 0067-2904

\title{
Separation Axioms in Topological Ordered Spaces Via b-open Sets
}

\author{
R. N. Majeed $^{1^{*}}$, S. A. El-Sheikh ${ }^{2}$ \\ ${ }^{1}$ Department of Mathematics, College of Education for Pure Science Ibn Al-Haitham, University of Baghdad, \\ Baghdad, Iraq \\ ${ }^{2}$ Department of Mathematics, Faculty of Education, Ain Shams University, Cairo, Egypt
}

Received: $18 / 8 / 2020$

Accepted: 9/11/2020

\begin{abstract}
This paper aims to define and study new separation axioms based on the b-open sets in topological ordered spaces, namely strong $b$ - $T_{i}$-ordered spaces $(i=0,1,2)$. These new separation axioms are lying between strong $T_{i}$-ordered spaces and $b-T_{i}$ spaces $(i=0,1,2)$. The implications of these new separation axioms among themselves and other existing types are studied, giving several examples and counterexamples. Also, several properties of these spaces are investigated; for example, we show that the property of strong $b$ - $T_{i}$-ordered spaces $(i=0,1,2)$ is an inherited property under open subspaces.
\end{abstract}

Keywords: topological ordered space, b-open set, increasing b-open set, decreasing b-open set, strong $b-T_{i}$-ordered space $(i=0,1,2)$

$$
\begin{aligned}
& \text { bايهيات الفصل في الفضاءات التبولوجية المرتبة بواسطة المجموعات المفتوحة-b }
\end{aligned}
$$

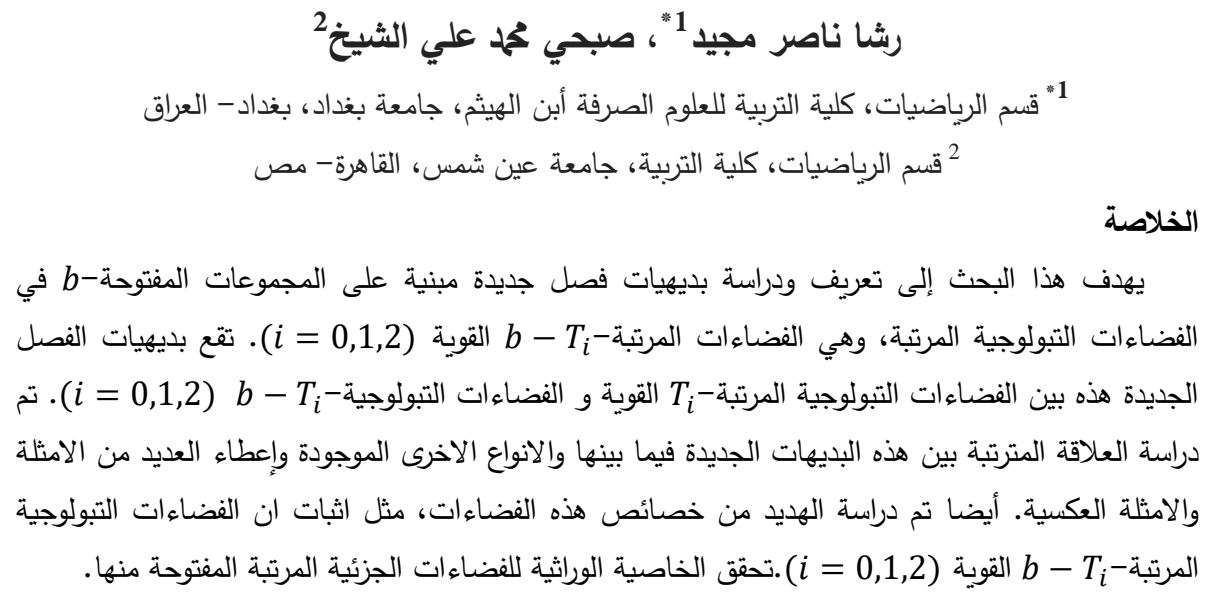

\section{1- Introduction}

In 1965, Nachbin [1] began the study of topological ordered spaces (Top-o.sp, for short) by associating a partially ordered relation with topological space. In 1968, McCartan [2] presented $\mathrm{T}_{\mathrm{i}^{-}}$ separation axioms $(i=1,2,3,4)$ inTop-o.sp's and explored several properties. He also acquired strong axioms by substituting the concept of the neighborhood with that of an open neighborhood. Arya and Gupta, in 1991, [3] presented and investigated some new separation axioms in Top-o.sp's, namely semi $\mathrm{T}_{1}$-ordered and semi $\mathrm{T}_{2}$-ordered. In 2002, Leela and Balasubramanian [4] used the notion of $\beta$ - 
open sets to introduce separation axioms in Top-o.sp's. Shanthi and Rajesh, in 2018, [5] introduced and studied some new types of separation axioms in Top-o.sp's via $\omega$-open sets. Recently, several researchers presented studies on Top-o.sp's $[6,7,8]$.

In the theme of general topology, generalized open sets play an important role and are currently considered as common research topics for many topologists worldwide. Indeed, by using generalized open sets, the significant themes of general topology and real analysis are concerned with the different modified forms of continuity, separation axioms, etc... [9, 10,11]. Another type of generalized open sets in topological space, presented by Andrijevic in 1996 [12], is the proposed b-open sets. This kind of sets was studied by Ekici and Caldas [13] under the name of $\gamma$-open sets. Also, Caldas and Jafari [14] and Park [15] used b-open sets to define $b-T_{i}(i=0,1)$ and $b-T_{2}$ separation axioms in topological spaces, respectively.

We, in this paper, present a new separation axiom in Top-o.sp, namely strong $b$ - $\mathrm{T}_{\mathrm{i}}$-ordered spaces $(i=0,1,2)$ and discuss some of their properties. Specifically, we show their relationships between

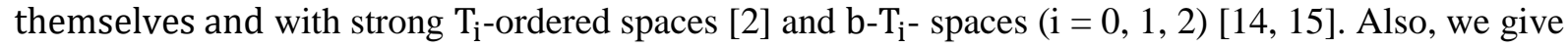
some characterizations of these new separation axioms and verify that the product of any family of strong $b-T_{i}$-ordered spaces is a strong $b-T_{i}$-ordered space $(i=0,1,2)$. Furthermore, we investigate the property of the strong $b-\mathrm{T}_{\mathrm{i}}$-ordered spaces $(\mathrm{i}=0,1)$ of being topological property under bijective, $\mathrm{b}$ open, and order reversing mappings, in addition to the property of the strong $b-T_{2}$-ordered space of being topological property under bijective, b-closed, and quotient order mappings.

\section{2- Preliminaries}

A Top-o.sp is a triple $(\mathrm{X}, \mathcal{T}, \mathcal{R})$, in which $(\mathrm{X}, \mathcal{T})$ is a topological space, and $(\mathrm{X}, \mathcal{R})$ is a poset. Through this paper, $\triangle$ refers to the diagonal relation on a non-empty set $X$.

Definition 2.1 [1]. Let $(\mathrm{X}, \mathcal{R})$ be a poset. Let $\mathrm{w} \in \mathrm{X}$ and $\mathcal{A} \subseteq \mathrm{X}$. Then

$1-[\mathrm{w},-\mathrm{]}]=\{\mathrm{z} \in \mathrm{X} ;(\mathrm{w}, \mathrm{z}) \in \mathcal{R}\}$,

2- $[-, \mathrm{w}]=\{\mathrm{z} \in \mathrm{X} ;(\mathrm{z}, \mathrm{w}) \in \mathcal{R}\}$,

3- $i(\mathcal{A})=\cup\{[a,-] ; a \in \mathcal{A}\}$,

4- $d(\mathcal{A})=\cup\{[-, a] ; a \in \mathcal{A}\}$,

5- $\mathcal{A}$ is called an increasing set ( $\mathcal{J}$-set, for short), if $\mathcal{A}=i(\mathcal{A})$,

6- $\mathcal{A}$ is called a decreasing set ( $\mathcal{D}$-set, for short), if $\mathcal{A}=d(\mathcal{A})$.

Definition 2.2 [2]. A Top-o.sp $(\mathrm{X}, \mathcal{T}, \mathcal{R})$ is called:

1- lower (resp. upper) strong $\mathrm{T}_{1}$-ordered (LS- $\mathrm{T}_{1}$-ordered (resp. US- $\mathrm{T}_{1}$-ordered), for short), if for each $\mathrm{w}, \mathrm{z} \in \mathrm{X}$, such that $(\mathrm{w}, \mathrm{z}) \notin \mathcal{R}$, there exists an $\mathcal{J}$ - (resp. an $\mathcal{D}$-) open set $\mathcal{U}$ of $\mathrm{w}$ (resp. z), such that $\mathrm{z}$ (resp. w) belongs to $\mathcal{U}^{\mathrm{c}}$.

2- strong $\mathrm{T}_{0}$-(resp. $\mathrm{T}_{1}$-)ordered ( $\mathrm{S}-\mathrm{T}_{0^{-}}$(resp. $\mathrm{S}-\mathrm{T}_{1}$-)ordered, for short), if it is an $\mathrm{LS}$ - $\mathrm{T}_{1}$-ordered or US$\mathrm{T}_{1}$-ordered (resp. LS- $\mathrm{T}_{1}$-ordered and US- $\mathrm{T}_{1}$-ordered).

3- strong $\mathrm{T}_{2}$-ordered ( $\mathrm{S}-\mathrm{T}_{2}$-ordered, for short), if for each $\mathrm{w}, \mathrm{z} \in \mathrm{X} ;(\mathrm{w}, \mathrm{z}) \notin \mathcal{R}$, there exist open sets $\mathcal{U}_{1}$ and $\mathcal{U}_{2}$ of $\mathrm{w}$ and $\mathrm{z}$, respectively, where $\mathcal{U}_{1}$ is an $\mathcal{J}$-set, $\mathcal{U}_{2}$ is an $\mathcal{D}$-set, and $\mathcal{U}_{1} \cap \mathcal{U}_{2}=\emptyset$.

Definition 2.3 [4]. Let $(X, \mathcal{T}, \mathcal{R})$ be a Top-o.sp. By the graph of the order of $\mathrm{X}$ we mean the subset of $\mathrm{X} \times \mathrm{X}$, namely $\{(\mathrm{w}, \mathrm{z}) \in \mathrm{X} \times \mathrm{X} ;(\mathrm{w}, \mathrm{z}) \in \mathcal{R}\}$.

Definition 2.4. Let $(\mathrm{X}, \mathcal{R})$ and $\left(\mathrm{Y}, \mathcal{R}^{*}\right)$ be two posets. Then, a mapping $\mathrm{F}:(\mathrm{X}, \mathcal{R}) \rightarrow\left(\mathrm{Y}, \mathcal{R}^{*}\right)$ is called:

1 - increasing, if for every $\mathrm{w}_{1}, \mathrm{w}_{2} \in \mathrm{X}$ such that $\left(\mathrm{w}_{1}, \mathrm{w}_{2}\right) \in \mathcal{R}$, then $\left(\mathrm{F}\left(\mathrm{w}_{1}\right), \mathrm{F}\left(\mathrm{w}_{2}\right)\right) \in \mathcal{R}^{*}[1]$.

2- decreasing, if for every $\mathrm{w}_{1}, \mathrm{w}_{2} \in \mathrm{X}$ such that $\left(\mathrm{w}_{1}, \mathrm{w}_{2}\right) \in \mathcal{R}$, then $\left(\mathrm{F}\left(\mathrm{w}_{2}\right), \mathrm{F}\left(\mathrm{w}_{1}\right)\right) \in \mathcal{R}^{*}[1]$.

3 - order-preserving, if for every $\mathrm{w}_{1}, \mathrm{w}_{2} \in \mathrm{X}$ such that $\left(\mathrm{w}_{1}, \mathrm{w}_{2}\right) \in \mathcal{R}$ iff $\left(\mathrm{F}\left(\mathrm{w}_{1}\right), \mathrm{F}\left(\mathrm{w}_{2}\right)\right) \in \mathcal{R}^{*}[16]$.

Definition 2.5 [17]. Let $(\mathrm{X}, \mathcal{R})$ and $\left(\mathrm{Y}, \mathcal{R}^{*}\right)$ be two posets and let $\mathrm{F}:(\mathrm{X}, \mathcal{R}) \rightarrow\left(\mathrm{Y}, \mathcal{R}^{*}\right)$ be a mapping, if $\mathrm{z}_{1}, \mathrm{z}_{2} \in \mathrm{Y},\left(\mathrm{z}_{1}, \mathrm{z}_{2}\right) \in \mathcal{R}^{*}$ and iff there exist $\mathrm{w}_{1}, \mathrm{w}_{2} \in \mathrm{X}, \mathrm{w}_{1} \in \mathrm{F}^{-1}\left(\mathrm{z}_{1}\right)$, and $\mathrm{w}_{2} \in \mathrm{F}^{-1}\left(\mathrm{z}_{2}\right)$ such that $\left(\mathrm{w}_{1}, \mathrm{w}_{2}\right) \in \mathcal{R}$. Then, $\mathcal{R}^{*}$ is named a quotient order of $\mathcal{R}$ induced by $\mathrm{F}$.

Definition 2.6 [12]. A subset $\mathcal{A}$ of a topological space $(\mathrm{X}, \mathcal{T})$ is said to be b-open set, if $\mathcal{A} \subset$ $\operatorname{int}(\operatorname{cl}(\mathcal{A})) \cup \operatorname{cl}(\operatorname{int}(\mathcal{A}))$. The complement of the b-open set is called the b-closed set. The class of all b-open sets in $\mathrm{X}$ will be denoted by $\mathrm{BO}(\mathrm{X})$.

Proposition 2.7 [12]

1. The union of any family of b-open sets is a b-open set.

2. The intersection of an open and a b-open set is a b-open set.

Definition 2.8 [14]. A space $(X, \mathcal{T})$ is said to be 
1 - $\mathrm{b}-\mathrm{T}_{0}$-space, if for any pair $\mathrm{w}, \mathrm{z}$ in $\mathrm{X}, \mathrm{w} \neq \mathrm{z}$, there is $\mathrm{a} \mathrm{b}$-open set $\mathcal{U}$ containing $\mathrm{w}$ but not $\mathrm{z}$ or $\mathrm{a} \mathrm{b}$ open set $\mathcal{V}$ containing $\mathrm{z}$ but not $\mathrm{w}$.

2- $\mathrm{b}-\mathrm{T}_{1}$-space, if for any pair $\mathrm{w}, \mathrm{z}$ in $\mathrm{X}, \mathrm{w} \neq \mathrm{z}$, there is $\mathrm{a} \mathrm{b}$-open set $\mathcal{U}$ containing $\mathrm{w}$ but not $\mathrm{z}$ and $\mathrm{a} \mathrm{b}$ open set $\mathcal{V}$ containing $\mathrm{z}$ but not $\mathrm{w}$.

3- $\mathrm{b}-\mathrm{T}_{2}$-space, if for any pair $\mathrm{w}, \mathrm{z}$ in $\mathrm{X}, \mathrm{w} \neq \mathrm{z}$, there is a b-open set $\mathcal{U}$ containing $\mathrm{w}$ and b-open set $\mathcal{V}$ containing z such that $\mathcal{U} \cap \mathcal{V}=\varnothing[15]$.

Definition 2.9 [13]. Let $(X, \mathcal{T})$ and $\left(Y, \mathcal{T}^{*}\right)$ be two topological spaces. Then, the mapping F: $(\mathrm{X}, \mathcal{T}) \rightarrow$ $\left(\mathrm{Y}, \mathcal{T}^{*}\right)$ is said to be

1 - b-continuous, if for each $w \in X$ and each open set $\mathcal{V}$ of $Y$ containing $F(w)$, there exists a b-open set $\mathcal{U}$ in $\mathrm{X}$ such that $\mathrm{F}(\mathcal{U}) \subseteq \mathcal{V}$.

2- b-irresolute, if for each b-open set $\mathcal{V}$ in $\mathrm{Y}, \mathrm{F}^{-1}(\mathcal{V})$ is a b-open set in $\mathrm{X}$.

3 - b-closed, if for each b-closed set $\mathcal{A}$ of $\mathrm{X}, \mathrm{F}(\mathcal{A})$ is a b-closed set in $\mathrm{Y}$.

4- b-open, if for each b-open set $\mathcal{A}$ of $\mathrm{X}, \mathrm{F}(\mathcal{A})$ is an open set in $\mathrm{Y}$.

3- Strong $b$ - $\mathbf{T}_{\mathbf{i}}$-ordered spaces, $\mathbf{i}=\mathbf{0}, \mathbf{1}$

Definition 3.1. A Top-o.sp $(\mathrm{X}, \mathcal{T}, \mathcal{R})$ is said to be

1- lower strong $\mathrm{b}-\mathrm{T}_{1}$-ordered space ( $\mathrm{LSb}-\mathrm{T}_{1}$-ordered $\mathrm{sp}$, for short), if for all $\mathrm{w}, \mathrm{z} \in \mathrm{X}$, such that $(\mathrm{w}, \mathrm{z}) \notin \mathcal{R}$, there is an $\mathcal{J}$-b-open set $\mathcal{U}$ such that $\mathrm{w} \in \mathcal{U}$ and $\mathrm{z} \notin \mathcal{U}$.

2- upper strong $\mathrm{b}$ - $\mathrm{T}_{1}$-ordered space (USb- $\mathrm{T}_{1}$-ordered $\mathrm{sp}$, for short), if for all $\mathrm{w}, \mathrm{z} \in \mathrm{X}$, such that $(\mathrm{w}, \mathrm{z}) \notin \mathcal{R}$, there is an $\mathcal{D}$-b-open set $\mathcal{V}$ such that $\mathrm{w} \notin \mathcal{V}$ and $\mathrm{z} \in \mathcal{V}$.

Definition 3.2. A Top-o.sp $(\mathrm{X}, \mathcal{T}, \mathcal{R})$ is said to be

1 - strong $\mathrm{b}-\mathrm{T}_{0}$-ordered space ( $\mathrm{Sb}-\mathrm{T}_{0}$-ordered $\mathrm{sp}$, for short) if it is an $\mathrm{LSb}^{-} \mathrm{T}_{1}$-ordered $\mathrm{sp}$ or USb- $\mathrm{T}_{1}$ ordered sp.

2- strong $\mathrm{b}$ - $\mathrm{T}_{1}$-ordered space (Sb- $\mathrm{T}_{1}$-ordered $\mathrm{sp}$, for short) if it is an $\mathrm{LSb}-\mathrm{T}_{1}$-ordered $\mathrm{sp}$ and $\mathrm{USb}-\mathrm{T}_{1^{-}}$ordered sp.

Clearly, from Definition 3.2, every strong $b-\mathrm{T}_{1}$-ordered space implies a strong $\mathrm{b}$ - $\mathrm{T}_{0}$-ordered space, while the opposite does not hold, as we explain in the next example.

Example 3.3. Let $\mathrm{X}=\{x, y, \mathrm{z}, \mathrm{w}\}, \tau=\{\mathrm{X}, \emptyset,\{x\},\{y\},\{x, y\}\}$, and $\mathcal{R}$ be the partial order relation on $\mathrm{X}$, defined as $\mathcal{R}=\triangle \mathrm{U}\{(x, y),(x, \mathrm{z}),(x, \mathrm{w})\}$. Then, $(\mathrm{X}, \mathcal{T}, \mathcal{R})$ is USb-T $\mathrm{T}_{1}$-ordered sp. But $(\mathrm{X}, \mathcal{T}, \mathcal{R})$ is not $\mathrm{LSb}$ - $\mathrm{T}_{1}$-ordered $\mathrm{sp}$, as $(\mathrm{z}, \mathcal{Y}) \notin \mathcal{R}$ and there does not exist an $\mathcal{J}$-b-open set $\mathcal{U}$ such that $\mathrm{z} \in \mathcal{U}$, $y \notin U$. Hence, $(\mathrm{X}, \mathcal{T}, \mathcal{R})$ is $\mathrm{Sb}-\mathrm{T}_{0}$-ordered sp and not $\mathrm{Sb}-\mathrm{T}_{1}$-ordered sp.

Proposition 3.4. Let $(\mathrm{X}, \mathcal{T}, \mathcal{R})$ be a Top-o.sp. Then,

1. $\mathrm{S}$ - $\mathrm{T}_{1}$-ordered $\mathrm{sp} \Rightarrow \mathrm{Sb}$ - $\mathrm{T}_{1}$-ordered $\mathrm{sp} \Rightarrow \mathrm{b}$ - $\mathrm{T}_{1}$-sp

2. $\mathrm{S}$ - $\mathrm{T}_{0}$-ordered $\mathrm{sp} \Rightarrow \mathrm{Sb}-\mathrm{T}_{0}$-ordered $\mathrm{sp} \Rightarrow \mathrm{b}-\mathrm{T}_{0}$-sp.

Proof. We prove part 1 only and, similarly, one can obtain part 2 . Since every open set is a b-open set [2], we have that every $\mathrm{S}-\mathrm{T}_{1}$-ordered $\mathrm{sp}$ is $\mathrm{Sb}$ - $\mathrm{T}_{1}$-ordered $\mathrm{sp}$. Now, suppose that $(\mathrm{X}, \mathcal{T}, \mathcal{R})$ is $\mathrm{Sb}-\mathrm{T}_{1}$ ordered sp and let $\mathrm{w}, \mathrm{z} \in \mathrm{X}$, such $\mathrm{w} \neq \mathrm{z}$. If $(\mathrm{w}, \mathrm{z}) \notin \mathcal{R}$, then from the hypothesis, we get the result straight and the proof is finished. If $\mathrm{w} \neq \mathrm{z}$ and $(\mathrm{w}, \mathrm{z}) \in \mathcal{R}$, then by the anti-symmetric condition of $\mathcal{R}$, it follows that $(\mathrm{z}, \mathrm{w}) \notin \mathcal{R}$ and, from the hypothesis, we get the result. Hence, $(\mathrm{X}, \mathcal{T}, \mathcal{R})$ is $\mathrm{b}$ - $\mathrm{T}_{1}$-ordered sp.

The converse of Proposition 3.4 does not hold, as the following examples explain.

In the first example, we show that $\mathrm{Sb}$ - $\mathrm{T}_{1}$-ordered sp does not imply $\mathrm{S}$ - $\mathrm{T}_{1}$-ordered $\mathrm{sp}$.

Example 3.5. Let $\mathrm{X}=\{x, y, \mathrm{z}\}, \tau=\{\mathrm{X}, \emptyset,\{x\},\{y\},\{x, y\}\}$, and $\mathcal{R}$ be the partial order relation on $\mathrm{X}$, defined as $\mathcal{R}=\Delta \mathrm{U}\{(\mathrm{z}, x)\}$. Then, $(\mathrm{X}, \mathcal{T}, \mathcal{R})$ is $\mathrm{Sb}$ - $\mathrm{T}_{1}$-ordered sp. However, it is not $\mathrm{S}$ - $\mathrm{T}_{1}$-ordered sp since it is not LS-T $\mathrm{T}_{1}$-ordered sp, as $(\mathrm{z}, \mathcal{Y}) \notin \mathcal{R}$ and there does not exist an $\mathcal{J}$-open set $\mathcal{U}$ such that $\mathrm{z} \in$ $\mathcal{u}, y \notin \mathcal{U}$.

Also, Example 3.3 shows $\mathrm{Sb}-\mathrm{T}_{0}$-ordered sp but not $\mathrm{S}$ - $\mathrm{T}_{0}$-ordered $\mathrm{sp}$, since it is not $\mathrm{LS}$ - $\mathrm{T}_{1}$-ordered $\mathrm{sp}$, as $(\mathrm{z}, \mathcal{Y}) \notin \mathcal{R}$ and there does not exist an $\mathcal{J}$-open set $\mathcal{U}$ such that $\mathrm{z} \in \mathcal{U}, \mathcal{y} \notin \mathcal{U}$.

Next, we need to introduce the notion of b-convergence to a point in topological spaces.

Definition 3.6. Let $(X, \mathcal{T})$ be a topological space. A net $\left\{k_{n}: n \in \mathfrak{D}\right\}$ in $X$ is said to be b-convergence to a point $\mathrm{w} \in \mathrm{X}$, if for every b-open set $\mathcal{U}$ contains $\mathrm{w}$, there exists $\mathrm{n} \in \mathfrak{D}$ such that for every $\mathrm{m} \in \mathfrak{D}$, $(\mathrm{n}, \mathrm{m}) \in \mathcal{R} \Rightarrow \mathrm{k}_{\mathrm{m}} \in \mathcal{U}$.

Theorem 3.7. Let $(\mathrm{X}, \mathcal{T}, \mathcal{R})$ be a Top-o.sp. Then, the followings are equivalent:

1 - $\quad(\mathrm{X}, \mathcal{T}, \mathcal{R})$ is $\mathrm{LSb}-\mathrm{T}_{1}$-ordered sp. 
2- For $\mathrm{w}, \mathrm{z} \in \mathrm{X}$ with $(\mathrm{w}, \mathrm{z}) \notin \mathcal{R}$, there is a b-open set $\mathcal{S}$ such that $\mathrm{w} \in \mathcal{S}$ and $(\mathrm{s}, \mathrm{z}) \notin \mathcal{R}$ for every $\mathrm{s} \in \mathcal{S}$.

3- For all $\mathrm{w} \in \mathrm{X},[-, \mathrm{w}]$ is a b-closed set.

4- If a net $\left\{\mathrm{w}_{\alpha}: \alpha \in \lambda\right\}$ is b-convergence to $\mathrm{w}$ and $\left(\mathrm{w}_{\alpha}, \mathrm{z}\right) \in \mathcal{R}$ for all $\alpha \in \lambda$, then $(\mathrm{w}, \mathrm{z}) \in \mathcal{R}$.

Proof. (1) $\Rightarrow(2)$. Let $\mathrm{w}, \mathrm{z} \in \mathrm{X}$ such that $(\mathrm{w}, \mathrm{z}) \notin \mathcal{R}$. Then, there exists an $\mathcal{J}$-b-open set $\mathcal{S}$ containing $\mathrm{w}$ and $\mathrm{z} \notin \mathcal{S}$. Let $\mathrm{s} \in \mathcal{S}$. If $(\mathrm{s}, \mathrm{z}) \in \mathcal{R}$, then $\mathrm{z} \in \mathcal{S}$ because $\mathcal{S}$ is an $\mathcal{J}$-set. So, (s, $\mathrm{z}) \notin \mathcal{R}$.

(2) $\Rightarrow(3)$. Let $\mathrm{w} \in \mathrm{X}$ and let $\mathrm{z} \in \mathrm{X}-[-, \mathrm{w}]$, then $\mathrm{z} \in \mathrm{X}$ and $\mathrm{z} \notin[-, \mathrm{w}]$, which means that $(\mathrm{z}, \mathrm{w}) \notin$ $\mathcal{R}$. Then, there exists by definition a b-open set $\mathcal{S}$ such that $\mathrm{z} \in \mathcal{S}, \mathrm{w} \notin \mathcal{S}$, and $(\mathrm{s}, \mathrm{w}) \notin \mathcal{R}$ for all $\mathrm{s} \in \mathcal{S}$ (i.e., $\mathrm{z} \in \mathcal{S} \subseteq \mathrm{X}-[-, \mathrm{w}])$. Hence, $\mathrm{X}-[-, \mathrm{w}]$ is a b-open set and consequently, $[-, \mathrm{w}]$ is a b-closed set.

(3) $\Rightarrow(1)$. Suppose that, for all $\mathrm{w} \in \mathrm{X},[-, \mathrm{w}]$ is a b-closed set. Let $\mathrm{w}, \mathrm{z} \in \mathrm{X}$ such that $(\mathrm{w}, \mathrm{z}) \notin \mathcal{R}$. From the hypothesis, $[-, \mathrm{z}]$ is a b-closed set and $\mathrm{w} \notin[-, \mathrm{z}]$, then $\mathrm{w} \in \mathrm{X}-[-, \mathrm{z}]=\mathcal{S}$ is a b-open set. This means that there exists $\mathcal{S}$ as a b-open set such that w $\in \mathcal{S}$ and $\mathrm{z} \notin \mathcal{S}$. Now, to show that $\mathcal{S}$ is an $\mathcal{J}$ set, let $\mathcal{S}=\mathrm{X}-[-, \mathrm{z}], \mathrm{s} \in \mathcal{S}$ and $\mathrm{q} \in[\mathrm{s},-]$. This implies that $(\mathrm{s}, \mathrm{z}) \notin \mathcal{R}$. If $\mathrm{q} \notin \mathcal{S}$, then $\mathrm{q} \in[-, \mathrm{z}]$, which yields $(\mathrm{q}, \mathrm{z}) \in \mathcal{R}$. Since $(\mathrm{s}, \mathrm{q}) \in \mathcal{R}$ and $(\mathrm{q}, \mathrm{z}) \in \mathcal{R}$, then by transitivity of $\mathcal{R}$, we get that $(\mathrm{s}, \mathrm{z}) \in \mathcal{R}$, implies $\mathrm{s} \in[-\mathrm{z}]$, which is a contradiction. Hence, $(\mathrm{q}, \mathrm{z}) \notin \mathcal{R}$ and then $\mathrm{q} \in \mathcal{S}$. Thus, $\mathcal{S}=i(\mathcal{S})$.

(3) $\Rightarrow(4)$. Let $\left\{\mathrm{w}_{\alpha}: \alpha \in \lambda\right\}$ be a net in $\mathrm{X}$, b-converging to a point $\mathrm{w}$, and let $\mathrm{z} \in \mathrm{X}$ such that $\left(\mathrm{w}_{\alpha}, \mathrm{z}\right) \in$ $\mathcal{R}$ for all $\alpha \in \lambda$. Suppose that $(\mathrm{w}, \mathrm{z}) \notin \mathcal{R}$ and, by $(3),[-, \mathrm{z}]$ is a b-closed set and $\mathrm{w} \notin[-, \mathrm{z}]$, then $\mathrm{w} \in \mathrm{X}-[-, \mathrm{z}]$, which is a b-open set containing w. Since $\left\{\mathrm{w}_{\alpha}: \alpha \in \lambda\right\}$ converges to $\mathrm{w}$, then there is $\mathrm{k} \in \lambda$ such that $\mathrm{w}_{\alpha} \in \mathrm{X}-[-, \mathrm{z}]$ for each $\alpha \geq \mathrm{k}$. That is, $\left(\mathrm{w}_{\alpha}, \mathrm{z}\right) \notin \mathcal{R}$ for all $\alpha \geq \mathrm{k}$, which is a contradiction.

(4) $\Rightarrow(2)$. Let $\left\{\mathrm{w}_{\alpha}: \alpha \in \lambda\right\}$ be a net, b-convergence to $\mathrm{w}$, and $\mathrm{z} \in \mathrm{X}$ such that $\left(\mathrm{w}_{\alpha}, \mathrm{z}\right) \in \mathcal{R}$ for all $\alpha \in \lambda$, then $(\mathrm{w}, \mathrm{z}) \in \mathcal{R}$. To prove (2), suppose that there exists $\mathrm{w}, \mathrm{z} \in \mathrm{X} ;(\mathrm{w}, \mathrm{z}) \notin \mathcal{R}$ such that for all bopen $\mathcal{S}$ containing $\mathrm{w},(\mathrm{s}, \mathrm{z}) \in \mathcal{R}$ for all $\mathrm{s} \in \mathcal{S}$. Let $\mathcal{S}$ be the collection for all b-open sets which contain $\mathrm{w}$, then $\mathcal{S}$ is a directed set by inclusion and the net $\{\mathrm{s}: \mathrm{s} \in \mathcal{S} \in \mathcal{S}\}$ is b-convergence to w. By hypothesis, $(\mathrm{w}, \mathrm{z}) \in \mathcal{R}$, and this is a contradiction.

Theorem 3.8. Let $(X, \mathcal{T}, \mathcal{R})$ be a Top-o.sp. Then, the followings are equivalent:

1 - $(\mathrm{X}, \mathcal{T}, \mathcal{R})$ is $\mathrm{USb}$ - $\mathrm{T}_{1}$-ordered sp.

2- For $\mathrm{w}, \mathrm{z} \in \mathrm{X}$ with $(\mathrm{w}, \mathrm{z}) \notin \mathcal{R}$, there is a b-open set $\mathcal{S}$ including $\mathrm{z}$ such that (w, s) $\notin \mathcal{R}$ for every $\mathrm{s} \in \mathcal{S}$.

3- For all $\mathrm{w} \in \mathrm{X},[\mathrm{w},-]$ is a b-closed set.

4- If there is a net $\left\{\mathrm{w}_{\alpha}: \alpha \in \lambda\right\}$ b-convergence to $\mathrm{w}$ and $\left(\mathrm{z}, \mathrm{w}_{\alpha}\right) \in \mathcal{R}$ for all $\alpha \in \lambda$, then $(\mathrm{z}, \mathrm{w}) \in \mathcal{R}$.

Proof. Similar to the proof of Theorem 3.7.

Corollary 3.9. A Top-o.sp $(\mathrm{X}, \mathcal{T}, \mathcal{R})$ is $\mathrm{Sb}-\mathrm{T}_{0}$-ordered $\mathrm{sp}$ if and only if either $[\mathrm{w},-]$ or $[-, \mathrm{w}]$ is a bclosed set for all $\mathrm{w} \in \mathrm{X}$.

Proof. Suppose that $(\mathrm{X}, \mathcal{T}, \mathcal{R})$ is $\mathrm{Sb}-\mathrm{T}_{0}$-ordered sp. Then, $\mathrm{X}$ is either $\mathrm{LSb}$ - $\mathrm{T}_{1}$-ordered sp or USb- $\mathrm{T}_{1}$ ordered sp. By Theorem 3.7(3), $[-, \mathrm{w}]$ is a b-closed set for all $\mathrm{w} \in \mathrm{X}$.

Conversely, if $[w,-]$ is b-closed set for all $w \in X$, then from Theorem $3.8((3) \Rightarrow(1))$, we have $X$ is USb- $\mathrm{T}_{1}$-ordered $\mathrm{sp}$, implies $\mathrm{X}$ is $\mathrm{Sb}$ - $\mathrm{T}_{0}$-ordered sp. Similarly, if $[-, \mathrm{w}]$ is $\mathrm{b}$-closed set for all $\mathrm{w} \in \mathrm{X}$, then from Theorem $3.7((3) \Rightarrow(1))$, we have $\mathrm{X}$ is LSb- $\mathrm{T}_{1}$-ordered sp, implies $\mathrm{X}$ is Sb- $\mathrm{T}_{0}$-ordered $\mathrm{sp}$.

Corollary 3.10. A Top-o.sp $(X, \mathcal{T}, \mathcal{R})$ is $S b-T_{1}$-ordered sp if and only if either $[w,-]$ or $[-, w]$ is a bclosed set for all $\mathrm{w} \in \mathrm{X}$.

Proof. The proof follows directly from the fact that every strong $\mathrm{b}-\mathrm{T}_{1}$-ordered space is strong $\mathrm{b}-\mathrm{T}_{0^{-}}$ ordered space, and from Corollary 3.9.

Remark 3.11. The property of being $S b-\mathrm{T}_{\mathrm{i}}$-ordered $\mathrm{sp}, \mathrm{i}=0,1$ is not a hereditary property. We display this by the two examples below.

Example 3.12. In Example 3.3, $(\mathrm{X}, \mathcal{T}, \mathcal{R})$ is $\mathrm{Sb}$ - $\mathrm{T}_{0}$-ordered sp. Consider that $\mathcal{A}=\{y, \mathrm{w}\} \subseteq \mathrm{X}$. Then we have $\mathcal{T}_{\mathcal{A}}=\{\mathcal{A}, \emptyset\}$ and $\mathcal{R}_{\mathcal{A}}=(\mathcal{A} \times \mathcal{A}) \cap \mathcal{R}=\{(y, y),(\mathrm{w}, \mathrm{w})\}$. Clearly, $(\mathrm{w}, y) \notin \mathcal{R}_{\mathcal{A}}$. However, there does not exist an $\mathcal{J}$-b-open set $\mathcal{U}$ such that w $\in \mathcal{U}$ and $\mathcal{y} \notin U$. This yield $\left(\mathcal{A}, \mathcal{T}_{\mathcal{A}}, \mathcal{R}_{\mathcal{A}}\right)$ is not

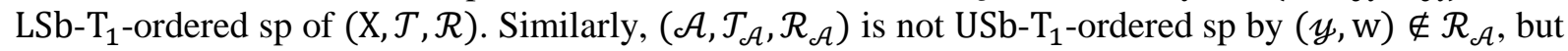
there does not exist an $\mathcal{D}$-b-open set $\mathcal{U}$ such that $\mathrm{w} \in \mathcal{U}$ and $\mathcal{y} \notin \mathcal{U}$. Therefore, $\left(\mathcal{A}, \mathcal{T}_{\mathcal{A}}, \mathcal{R}_{\mathcal{A}}\right)$ is not $\mathrm{Sb}$ $\mathrm{T}_{0}$-ordered subsp of $(\mathrm{X}, \mathcal{T}, \mathcal{R})$. 
Example 3.13. Let $X=\{x, y, z\}, \tau=\{X, \emptyset,\{z\},\{y\},\{z, y\}\}$, and $\mathcal{R}$ be the partial order relation on $X$ defined as $\mathcal{R}=\triangle \mathrm{U}\{(x, y),(x, \mathrm{z})\}$. Then, $(\mathrm{X}, \mathcal{T}, \mathcal{R})$ is Sb-T -ordered sp. Consider that $\mathcal{A}=\{x, \mathrm{z}\} \subseteq$ $\mathrm{X}$, this implies that $\mathcal{T}_{\mathcal{A}}=\{\mathcal{A}, \emptyset,\{\mathrm{z}\}\}$ and $\mathcal{R}_{\mathcal{A}}=(\mathcal{A} \times \mathcal{A}) \cap \mathcal{R}=\{(x, x),(x, \mathrm{z}),(\mathrm{z}, \mathrm{z})\}$. Thus, $\left(\mathcal{A}, \mathcal{T}_{\mathcal{A}}, \mathcal{R}_{\mathcal{A}}\right)$ is an ordered subsp of $(\mathrm{X}, \mathcal{T}, \mathcal{R})$ which is not USb-T $\mathrm{T}_{1}$-ordered sp, since $(\mathrm{z}, x) \notin \mathcal{R}_{\mathcal{A}}$ and there does not exist an $\mathcal{D}$-b-open set $\mathcal{U}$ such that $x \in \mathcal{U}$ and z $\notin U \mathcal{U}$. Therefore, $\left(\mathcal{A}, \mathcal{T}_{\mathcal{A}}, \mathcal{R}_{\mathcal{A}}\right)$ is not Sb$\mathrm{T}_{1}$-ordered subsp of $(\mathrm{X}, \mathcal{T}, \mathcal{R})$.

The next theorem provides the condition that satisfies the hereditary property in $\mathrm{Sb}$ - $\mathrm{T}_{\mathrm{i}}$-ordered $\mathrm{sp}$, $\mathrm{i}=0,1$.

Theorem 3.14. Every open subspace of an $S b-T_{i}$-ordered $s p$ is $S b-T_{i}$-ordered $s p, i=0,1$.

Proof. We shall prove the theorem for $\mathrm{i}=1$ only. Let $(\mathrm{X}, \mathcal{T}, \mathcal{R})$ be an $\mathrm{Sb}-\mathrm{T}_{1}$-ordered sp and $\mathcal{A}$ is an open subspace of $\mathrm{X}$. Let $\mathrm{w} \in \mathcal{A}$, then $\mathrm{w} \in \mathrm{X}$. Since $\mathrm{X}$ is $\mathrm{Sb}-\mathrm{T}_{1}$-ordered $\mathrm{sp}$, then by Theorem 3.7 and Theorem $3.8((1) \Rightarrow(3))$, we have $[\mathrm{w},-]]$ and $[-, \mathrm{w}]$ are b-closed sets in $\mathrm{X}$. This implies that $\mathrm{X}-[\mathrm{w},-]$ and $\mathrm{X}-[-, \mathrm{w}]$ are b-open sets in X. Since $\mathcal{A}$ is an open subset of $\mathrm{X}$, then by Proposition 2.3(2), we get $\mathcal{A} \cap(\mathrm{X}-[\mathrm{w},-])$ and $\mathcal{A} \cap(\mathrm{X}-[-, \mathrm{w}])$ are b-open sets in $\mathrm{X}$. But $\mathcal{A} \cap(\mathrm{X}-$ $[\mathrm{w},-])=\mathcal{A} \cap(\{\mathrm{X}-\{\mathrm{z} \in \mathrm{X}:(\mathrm{w}, \mathrm{z}) \in \mathcal{R}\}\})=\mathcal{A}-\{\mathrm{z} \in \mathrm{X}:(\mathrm{w}, \mathrm{z}) \in \mathcal{R}\}=\mathcal{A}-[\mathrm{w},-]]$ is b-open set and $[\mathrm{w},-]$ and $[-, \mathrm{w}]$ are b-closed sets. By (3) $\Rightarrow$ (1) of Theorem 3.7 and Theorem 3.8, it follows that $\left(\mathcal{A}, \mathcal{T}_{\mathcal{A}}, \mathcal{R}_{\mathcal{A}}\right)$ is $\mathrm{Sb}-\mathrm{T}_{1}$-ordered sp.

Theorem 3.15 A Top-o.sp $(X, \mathcal{T}, \mathcal{R})$ is $S b-T_{1}$-ordered sp if and only if for all $w \in X$, there exists a $S b$ $T_{1}$-ordered $b$-open set in $X$ that is $\mathcal{J}$ - and $\mathcal{D}$-set containing $w$.

Proof. Suppose that $(X, \mathcal{T}, \mathcal{R})$ is $S b-T_{1}$-ordered sp. Then, for each $w \in X, X$ is the required set.

Conversely, suppose that for all $\mathrm{w} \in \mathrm{X}$, there is an Sb-T $\mathrm{T}_{1}$-ordered b-open set $\mathcal{S}_{1}$ in X which is $\mathcal{J}$ - and $\mathcal{D}$ - and $\mathrm{w} \in \mathcal{S}_{1}$ for all $\mathrm{w}$. Let $(\mathrm{w}, \mathrm{z}) \notin \mathcal{R}$. Then, by the hypothesis, there exist $\mathrm{Sb}$ - $\mathrm{T}_{1}$-ordered b-open sets $\mathcal{S}_{1}$ and $\mathcal{S}_{2}$ in X containing $\mathrm{w}$ and $\mathrm{z}$, respectively, where $\mathcal{S}_{1}$ and $\mathcal{S}_{2}$ are both $\mathcal{J}$ - and $\mathcal{D}$-sets. If z $\notin \mathcal{S}_{1}$ and $\mathrm{w} \notin \mathcal{S}_{2}$, then there is nothing to prove. But, if $\mathrm{z} \in \mathcal{S}_{1}, \mathrm{w} \in \mathcal{S}_{1}$, and $(\mathrm{w}, \mathrm{z}) \notin \mathcal{R}$ and if $\mathcal{S}_{1}$ is $\mathrm{Sb}-\mathrm{T}_{1}$ ordered sp, this implies that there exists $\mathcal{J}$-b-open set $\mathcal{V}_{1}$ such that $\mathcal{V}_{1} \subseteq \mathcal{S}_{1} \subseteq \mathrm{X}, \mathrm{w} \in \mathcal{V}_{1}$, and $\mathrm{z} \notin \mathcal{V}_{1}$. Also, if $\mathrm{z} \in \mathcal{S}_{2}, \mathrm{w} \in \mathcal{S}_{2}$, and $(\mathrm{w}, \mathrm{z}) \notin \mathcal{R}$, and if $\mathcal{S}_{2}$ is $\mathrm{Sb}$ - $\mathrm{T}_{1}$-ordered $\mathrm{sp}$, this implies that there exists $\mathcal{D}$ b-open set $\mathcal{V}_{2}$ such that $\mathcal{V}_{2} \subseteq \mathcal{S}_{2} \subseteq \mathrm{X}, \mathrm{z} \in \mathcal{V}_{2}$, and w $\notin \mathcal{V}_{2}$. Hence, $(\mathrm{X}, \mathcal{T}, \mathcal{R})$ is $\mathrm{Sb}$ - $\mathrm{T}_{1}$-ordered sp.

Theorem 3.16. Let $\mathrm{F}$ be an order-preserving $b$-open mapping from a Top-o.sp $(X, \mathcal{T}, \mathcal{R})$ onto a Topo.sp $\left(Y, \mathcal{T}^{*}, \mathcal{R}^{*}\right)$. If $X$ is $S b-T_{1}$-ordered sp, then $Y$ is also so.

Proof. Let $\mathrm{z}_{1}, \mathrm{z}_{2} \in \mathrm{Y}$ such that $\left(\mathrm{z}_{1}, \mathrm{z}_{2}\right) \notin \mathcal{R}^{*}$. Since $\mathrm{F}$ is onto, then there exist $\mathrm{w}_{1}, \mathrm{w}_{2} \in \mathrm{X}$ such that $\mathrm{F}\left(\mathrm{w}_{1}\right)=\mathrm{z}_{1}, \mathrm{~F}\left(\mathrm{w}_{2}\right)=\mathrm{z}_{2}$ and, by order-preserving mapping, we have $\left(\mathrm{w}_{1}, \mathrm{w}_{2}\right) \notin \mathcal{R}$. Since $(\mathrm{X}, \mathcal{T}, \mathcal{R})$ is $\mathrm{Sb}$ - $\mathrm{T}_{1}$-ordered sp, then there exists an $\mathcal{J}$-b-open set $\delta_{1}$ such that $\mathrm{w}_{1} \in \mathcal{S}_{1}, \mathrm{w}_{2} \notin \mathcal{S}_{1}$ and there exists an $\mathcal{D}$-b-open set $\mathcal{S}_{2}$ such that $\mathrm{w}_{2} \in \mathcal{S}_{2}, \mathrm{w}_{1} \notin \mathcal{S}_{2}$. By $\mathrm{F}$ is b-open mapping, then $\mathrm{F}\left(\mathcal{S}_{1}\right), \mathrm{F}\left(\mathcal{S}_{2}\right)$ are b-open sets in $\mathrm{Y}$ and there exists an $\mathcal{J}$-b-open set $\mathrm{F}\left(\mathcal{S}_{1}\right)$ containing $\mathrm{z}_{1}$, not $\mathrm{z}_{2}$, and $\mathcal{D}$-b-open set $\mathrm{F}\left(\mathcal{S}_{2}\right)$ containing $\mathrm{z}_{2}$, not $\mathrm{z}_{1}$. Hence, $\left(\mathrm{Y}, \mathcal{T}^{*}, \mathcal{R}^{*}\right)$ is $\mathrm{Sb}-\mathrm{T}_{1}$-ordered sp.

Corollary 3.17. The property of being $S b-T_{0}$-ordered sp is preserved by onto, $b$-open, and orderpreserving mappings.

Theorem 3.18. Let $\mathrm{F}$ be an order-preserving b-irresolute mapping from a Top-o.sp $(\mathrm{X}, \mathcal{T}, \mathcal{R})$ to a Topo.sp $\left(\mathrm{Y}, \mathcal{T}^{*}, \mathcal{R}^{*}\right)$. If $\mathrm{Y}$ is $\mathrm{Sb}-\mathrm{T}_{1}$-ordered $\mathrm{sp}$, then $\mathrm{X}$ is also so.

Proof. Let $w_{1}, w_{2} \in X$ such that $\left(w_{1}, w_{2}\right) \notin \mathcal{R}$. Then, by $\mathrm{F}$ is order-preserving, we get $\left(\mathrm{F}\left(w_{1}\right), \mathrm{F}\left(w_{2}\right)\right) \notin \mathcal{R}^{*}$. Since $Y$ is $S b$ - $T_{1}$-ordered sp, then there exists an $\mathcal{J}$ - $b$-open set $\mathcal{K}_{1}$ such that $\mathrm{F}\left(w_{1}\right) \in \mathcal{K}_{1}, \mathrm{~F}\left(w_{2}\right) \notin \mathcal{K}_{1}$. By $\mathrm{F}$ is order-preserving $b$-irresolute mapping, we get $\mathrm{F}^{-1}\left(\mathcal{K}_{1}\right)=\mathcal{S}_{1}$ is an $\mathcal{J}$ - $b$-open set in $X$ such that $w_{1} \in \mathcal{S}_{1}, w_{2} \notin \mathcal{S}_{1}$. Thus, $(X, \mathcal{T}, \mathcal{R})$ is $L S b$ - $T_{1}$-ordered sp. Similarly, $(X, \mathcal{T}, \mathcal{R})$ is $U S b$ - $T_{1}$-ordered sp. Therefore, $(X, \mathcal{T}, \mathcal{R})$ is $S b$ - $T_{1}$-ordered sp. $\square$

Corollary 3.19. Let $\mathrm{F}$ be an order-preserving b-irresolute mapping from a Top-o.sp $(\mathrm{X}, \mathcal{T}, \mathcal{R})$ to a Top-o.sp $\left(\mathrm{Y}, \mathcal{T}^{*}, \mathcal{R}^{*}\right)$. If $\mathrm{Y}$ is $\mathrm{Sb}-\mathrm{T}_{0}$-ordered sp, then $\mathrm{X}$ is also so.

Theorem 3.20. Let $\left\{\left(X_{\alpha}, \mathcal{T}_{\alpha}, \mathcal{R}_{\alpha}\right): \alpha \in J\right\}$ be a family of $S b$ - $T_{i}$-ordered sp's. Then, the product of $\left\{\left(X_{\alpha}, \mathcal{T}_{\alpha}, \mathcal{R}_{\alpha}\right): \alpha \in J\right\}$ is also $S b$ - $T_{i}$-ordered sp, $i=0,1$.

Proof. We shall prove the theorem for $\mathrm{i}=1$ only. Let $(\mathrm{X}, \mathcal{T}, \mathcal{R})$ be the product ordered space. Let $\mathrm{w}=\left(\mathrm{w}_{\alpha}\right)$ and $\mathrm{z}=\left(\mathrm{z}_{\alpha}\right)$ be two elements of $\mathrm{X}$ such that $(\mathrm{w}, \mathrm{z}) \notin \mathcal{R}$. Thus, there exists $\mathrm{j} \in \mathrm{J}$ such that $\left(\mathrm{w}_{\mathrm{j}}, \mathrm{z}_{\mathrm{j}}\right) \notin \mathcal{R}_{\mathrm{j}}$. Since $\left(\mathrm{X}_{\mathrm{j}}, \mathcal{T}_{\mathrm{j}}, \mathcal{R}_{\mathrm{j}}\right)$ is also Sb-T $\mathrm{T}_{1}$-ordered sp, therefore there exists an $\mathcal{J}$ - $\mathcal{T}_{\mathrm{j}}$-b-open set $\mathcal{S}_{\mathrm{j}}$ such that $\mathrm{w}_{\mathrm{j}} \in \mathcal{S}_{\mathrm{j}}$ and $\mathrm{z}_{\mathrm{j}} \notin \mathcal{S}_{\mathrm{j}}$. We define $\mathcal{S}=\prod_{\alpha \in \mathrm{J}} \mathcal{S}_{\alpha}$, where $\mathcal{S}_{\alpha}=\mathrm{X}_{\alpha}$ if $\alpha \neq \mathrm{j}$. Then, $\mathcal{S}$ is an J-b-open 
set of $\mathrm{X}$ containing w but not $\mathrm{z}$. Therefore, $(\mathrm{X}, \mathcal{T}, \mathcal{R})$ is $\mathrm{LSb}-\mathrm{T}_{1}$-ordered sp. Similarly, it is also USb- $\mathrm{T}_{1}$ ordered sp. Hence, it is $\mathrm{Sb}-\mathrm{T}_{1}$-ordered sp.

Definition 3.21. A Top-o.sp $(\mathrm{X}, \mathcal{T}, \mathcal{R})$ is called $\mathrm{C}_{\mathrm{b}}$-space, if every b-closed set $\mathcal{F}$ of $\mathrm{X}, i(\mathcal{F})$, and $d(\mathcal{F})$ is b-closed set of X.

Next, we introduce some properties about $b-T_{1}$-sp, which is necessary to the proof of Theorem 3.25.

Proposition 3.22. A Top-o.sp $(X, \mathcal{T}, \mathcal{R})$ is $b-T_{1}$-sp if and only if $\{w\}$ is a b-closed set for each $w \in X$.

Proof. Suppose that $X$ is $b-T_{1}$-sp and $w \in X$. Let $z \notin X-\{w\}$. Then, $w \neq z$. Since $X$ is $b-T_{1}$-sp, then there exist b-open sets $\mathcal{U}, \mathcal{V}_{\mathrm{z}}$ such that $\mathrm{w} \in \mathcal{U}, \mathrm{z} \in \mathcal{V}_{\mathrm{z}}, \mathrm{z} \notin \mathcal{U}$, and $\mathrm{w} \notin \mathcal{V}_{\mathrm{z}}$. This means that $\mathrm{X}-\{\mathrm{w}\}$ is a $\mathrm{b}$-nbhd for all $\mathrm{z} \in \mathrm{X}-\{\mathrm{w}\}$. Thus, $\mathrm{X}-\{\mathrm{w}\}$ is b-open set and $\{\mathrm{w}\}$ is b-closed set.

Conversely, suppose that $\{w\}$ is $b$-closed set for each $w \in X$. Let $w \neq z$ in $X$. From the hypothesis, $\{w\},\{z\}$ are $b$-closed sets and $w \notin X-\{z\}, z \notin X-\{w\}$ are $b$-open sets. Hence, we get $X$ is $b-T_{1}$-sp.

Every $\mathrm{Sb}-\mathrm{T}_{1}$-o.sp $(\mathrm{X}, \mathcal{T}, \mathcal{R})$ is $\mathrm{b}-\mathrm{T}_{1}$-sp, but the converse might not hold. The next proposition gives the condition in which the converse holds.

Proposition 3.23. Let $(X, \mathcal{T}, \mathcal{R})$ be a $b-T_{1}$-sp and $C_{b}$-space. Then, it is $S b$ - $T_{1}$-ordered sp.

Proof. Let $w \in X$. By $X$ is $b-T_{1}$-sp, we get $\{w\}$ is $b$-closed for each $w \in X$. Also, from $X$ is $C_{b}$-space, then $i(\{w\})$ and $d(\{w\})$ are $b$-closed subsets of $X$. From Theorem 3.7((3) $\Rightarrow(1)),(X, \mathcal{T}, \mathcal{R})$ is $S b-T_{1}$ ordered sp.

Proposition 3.24. Let $\mathrm{F}:(\mathrm{X}, \mathcal{T}) \rightarrow\left(\mathrm{Y}, \mathcal{T}^{*}\right)$ onto, b-closed, and $\mathrm{X}$ is $\mathrm{b}-\mathrm{T}_{1}$-sp, then $\mathrm{Y}$ is $\mathrm{b}-\mathrm{T}_{1}$-sp.

Proof. Let $z \in Y$. Since $F$ is onto, then there exists $w \in X$ such that $F(w)=z$. Since $X$ is $b-T_{1}$-sp, then $\{w\}$ is b-closed and, by $F$ is b-closed mapping, we obtain $F(\{w\})=\{z\}$ is b-closed. Hence, $Y$ is $b-T_{1}$ sp.

Theorem 3.25. Let $\mathrm{F}:(\mathrm{X}, \mathcal{T}, \mathcal{R}) \rightarrow\left(\mathrm{Y}, \mathcal{T}^{*}, \mathcal{R}^{*}\right)$ be a b-irresolute and b-closed mapping from a $\mathrm{C}_{\mathrm{b}}$ - Sb$\mathrm{T}_{1}$-ordered sp $(\mathrm{X}, \mathcal{T}, \mathcal{R})$ onto $\left(\mathrm{Y}, \mathcal{T}^{*}, \mathcal{R}^{*}\right)$, where $\mathcal{R}^{*}$ is the quotient order of $\mathcal{R}$ induced by $\mathrm{F}$. Then, $\left(\mathrm{Y}, \mathcal{T}^{*}, \mathcal{R}^{*}\right)$ is $\mathrm{Sb}$ - $\mathrm{T}_{1}$-ordered sp.

Proof. Since $(\mathrm{X}, \mathcal{T}, \mathcal{R})$ is $\mathrm{Sb}-\mathrm{T}_{1}$-ordered sp, then $\mathrm{X}$ is $\mathrm{b}-\mathrm{T}_{1}$-sp. Since $\mathrm{F}$ is onto and $\mathrm{b}$-closed, then from Proposition 3. 24, $\mathrm{Y}$ is b- $\mathrm{T}_{1}$-sp. Let $\mathcal{F}^{*}$ be a b-closed set in $\mathrm{Y}$ and $\mathrm{F}$ is a b-irresolute, then $\mathrm{F}^{-1}\left(\mathcal{F}^{*}\right)$ is bclosed in $\mathrm{X}$. Since $\mathrm{X}$ is $\mathrm{C}_{\mathrm{b}}$-space, then $i\left(\mathrm{~F}^{-1}\left(\mathcal{F}^{*}\right)\right)$ and $d\left(\mathrm{~F}^{-1}\left(\mathcal{F}^{*}\right)\right)$ are b-closed subsets in $\mathrm{X}$. Since $\mathrm{F}$ is b-closed, then $\mathrm{F}\left(i\left(\mathrm{~F}^{-1}\left(\mathcal{F}^{*}\right)\right)\right)=i\left(\mathcal{F}^{*}\right)$ and $\mathrm{F}\left(d\left(\mathrm{~F}^{-1}\left(\mathcal{F}^{*}\right)\right)\right)=d\left(\mathcal{F}^{*}\right)$ are b-closed sets in Y. Hence, $\mathrm{Y}$ is $\mathrm{C}_{\mathrm{b}}$-space. Now, $\mathrm{Y}$ is $\mathrm{C}_{\mathrm{b}}$-space and $\mathrm{b}$ - $\mathrm{T}_{1}$-sp and, by Proposition 3.23, $\mathrm{Y}$ is $\mathrm{Sb}$ - $\mathrm{T}_{1}$-ordered sp. 4- Strong $b$ - $T_{2}$-ordered spaces

Definition 4.1. A Top-o.sp $(\mathrm{X}, \mathcal{T}, \mathcal{R})$ is called a strong $\mathrm{b}$ - $\mathrm{T}_{2}$-ordered space $\left(\mathrm{Sb}\right.$ - $\mathrm{T}_{2}$-ordered $\mathrm{sp}$, for short), if for each pair of elements $\mathrm{w}, \mathrm{z}$ such that $(\mathrm{w}, \mathrm{z}) \notin \mathcal{R}$ in $\mathrm{X}$, there are b-open sets $\mathcal{S}$ and $\mathcal{K}$ in $\mathrm{X}$ such that w $\in \mathcal{S}, \mathrm{z} \in \mathcal{K}$, and $\mathcal{S} \cap \mathcal{K}=\emptyset$, where $\mathcal{S}$ is $\mathcal{J}$-set and $\mathcal{K}$ is $\mathcal{D}$-set.

Proposition. 4.2 Let $(X, \mathcal{T}, \mathcal{R})$ be a Top-o.sp. Then,

$S$ - $T_{2}$-ordered $\mathrm{sp} \Rightarrow S b-T_{2}$-ordered $\mathrm{sp} \Rightarrow b-T_{2}$-sp

Proof. The proof that every $\mathrm{S}-\mathrm{T}_{2}$-ordered $\mathrm{sp}$ is $\mathrm{Sb}-\mathrm{T}_{2}$-ordered $\mathrm{sp}$ follows directly from the fact that every open set is b-open set. Now, suppose that $(\mathrm{X}, \mathcal{T}, \mathcal{R})$ is $\mathrm{Sb}-\mathrm{T}_{2}$-ordered sp and let $\mathrm{w}, \mathrm{z} \in \mathrm{X}$ such that $\mathrm{w} \neq \mathrm{z}$. If $(\mathrm{w}, \mathrm{z}) \notin \mathcal{R}$, then from hypothesis, we get the result directly and the proof is finished. If $\mathrm{w} \neq \mathrm{z}$ and $(\mathrm{w}, \mathrm{z}) \in \mathcal{R}$, then by the anti-symmetric property of $\mathcal{R}$, it follows that $(\mathrm{z}, \mathrm{w}) \notin \mathcal{R}$ and from hypothesis, we get the result. Hence, $(\mathrm{X}, \mathcal{T}, \mathcal{R})$ is $\mathrm{b}-\mathrm{T}_{2}$-ordered sp.

The reverse of the implications in Proposition 4.2 does not hold in general. Example 3.5 shows that $S b-T_{2}$-ordered sp does not imply to $S$ - $T_{2}$-ordered sp since there exists $(x, z) \notin \mathcal{R}$; however, disjoint open sets $\mathcal{S}$ and $\mathcal{K}$ do not exist in $X$ containing $x$ and $z$, respectively, where $\mathcal{S}$ is $\mathcal{J}$-set and $\mathcal{K}$ is $\mathcal{D}$-set.

Example 4.3. Let $\mathrm{X}=\{x, y, \mathrm{z}\}, \tau=\{\mathrm{X}, \emptyset,\{x\},\{\mathrm{z}\},\{x, \mathrm{z}\}\}$, and $\mathcal{R}$ be the partial order relation on $\mathrm{X}$, defined as $\mathcal{R}=\triangle \mathrm{U}\{(x, \mathrm{z})\}$. Then, $(\mathrm{X}, \mathcal{T}, \mathcal{R})$ is $\mathrm{b}-\mathrm{T}_{2}$-ordered sp. However, $(\mathrm{X}, \mathcal{T}, \mathcal{R})$ is not $\mathrm{Sb}-\mathrm{T}_{2}-$ ordered sp because there exists $(x, y) \notin \mathcal{R}$ and there do not exist disjoint b-open sets $\mathcal{S}$ and $\mathcal{K}$ in $\mathrm{X}$ containing $x$ and $\mathcal{y}$, respectively, where $\mathcal{S}$ is $\mathcal{J}$-set and $\mathcal{K}$ is $\mathcal{D}$-set.

Some characterizations of $S b-T_{2}$-ordered sp's will be given. But before that, we need to introduce the notion of increasing (resp. decreasing) $b$-nbhds.

Definition 4.4. A subset $\mathcal{A}$ of a Top-o.sp $(\mathrm{X}, \mathcal{T}, \mathcal{R})$ is said to be $\mathcal{J}$-b-nbhd (resp. $\mathcal{D}$-b-nbhd) of w in X, if there exists an $\mathcal{J}$ - (resp. $\mathcal{D}$-) b-open set $\mathcal{S}$ such that w $\in \mathcal{S} \subseteq \mathcal{A}$. 
Theorem 4.5. A Top-o.sp $(\mathrm{X}, \mathcal{T}, \mathcal{R})$ is $\mathrm{Sb}-\mathrm{T}_{2}$-ordered $\mathrm{sp}$ if and only if, for each $\mathrm{w} \in \mathrm{X}$, the intersection of all increasing (resp. decreasing) b-closed $\mathcal{J}$-b-nbhds (resp. $\mathcal{D}$-b-nbhds) of $\mathrm{w}$ is $[\mathrm{w},-\rightarrow]$ (resp. [$, \mathrm{w}]$ ).

Proof. Suppose that $(\mathrm{X}, \mathcal{T}, \mathcal{R})$ is $\mathrm{Sb}-\mathrm{T}_{2}$-ordered sp. Let $\mathrm{w} \in \mathrm{X}$. To prove that $[\mathrm{w},-]=\cap\{\mathcal{A}: \mathcal{A}$ is an $\mathcal{J}$-b-closed set $\mathcal{J}$-b-nbhd of $\mathrm{w}\}$, let $\mathcal{U}=\cap\{\mathcal{A}: \mathcal{A}$ is an $\mathcal{J}$-b-closed set $\mathcal{J}$-b-nbhd of $\mathrm{w}\}, \mathrm{W} \subseteq \mathcal{U}$. So, it we only need to prove that $\mathcal{U} \subseteq[\mathrm{w},-]$. Let $\mathrm{z} \notin[\mathrm{w},-]$. Then, $(\mathrm{w}, \mathrm{z}) \notin \mathcal{R}$. Since $\mathrm{X}$ is $\mathrm{Sb}-\mathrm{T}_{2}$-ordered $\mathrm{sp}$, there exists an $\mathcal{J}$-b-open set $\mathcal{S}$ containing $\mathrm{w}$ and $\mathcal{D}$-b-open set $\mathcal{K}$ containing $\mathrm{z}$ and $\mathcal{S} \cap \mathcal{K}=\emptyset$. Then, $\mathrm{w} \in \mathcal{S} \subseteq \mathcal{K}^{\mathrm{c}}$. Hence, $\mathcal{K}^{\mathrm{c}}$ is $\mathcal{J}$-b-closed set $\mathcal{J}$-b-nbhd of $\mathrm{w}, \mathrm{z} \notin \mathcal{K}^{\mathrm{c}}$. Then, $\mathrm{z} \notin \mathcal{U}$ and $\mathcal{U} \subseteq[\mathrm{w},-\rightarrow]$. Hence, $\mathcal{U}=[\mathrm{w},-]$. Similarly, we can demonstrate that $[-, \mathrm{w}]$ is the intersection of all $\mathcal{D}$-b-closed $\mathcal{D}$ b-nbhds of w.

Conversely, let $\mathrm{w}, \mathrm{z} \in \mathrm{X},(\mathrm{w}, \mathrm{z}) \notin \mathcal{R}$. Then $\mathrm{z} \notin[\mathrm{w},-]$ and consequently, there exists an J-b-closed set J-b-nbhd $\mathcal{A}$ of w such that $\mathrm{z} \notin \mathcal{A}$. It follows that $\mathrm{z} \in \mathcal{A}^{\mathrm{c}}$. $\mathcal{A}^{\mathrm{c}}$ is an $\mathcal{D}$-b-open set containing z. Also, there exists an $\mathcal{J}$-b-open set $\mathcal{K}$ such that $\mathrm{w} \in \mathcal{K} \subseteq \mathcal{A}$. $\mathcal{K} \cap \mathcal{A}^{\mathrm{c}}=\emptyset$. Hence, $(\mathrm{X}, \mathcal{T}, \mathcal{R})$ is $\mathrm{Sb}$ - $\mathrm{T}_{2}$-ordered sp. Similarly, we can show that $(\mathrm{X}, \mathcal{T}, \mathcal{R})$ is $\mathrm{Sb}-\mathrm{T}_{2}$-ordered sp if $[-, \mathrm{w}]$ is the intersection of all $\mathcal{D}$-bclosed $\mathcal{D}$-b-nbhds of $\mathrm{w}$ for all $\mathrm{w} \in \mathrm{X}$.

Theorem 4.6. A Top-o.sp $(\mathrm{X}, \mathcal{T}, \mathcal{R})$ is $\mathrm{Sb}-\mathrm{T}_{2}$-ordered sp if and only if for any $\mathrm{w} \in \mathrm{X}$, there is an $\mathcal{J}$ (resp. $\mathcal{D}$-) b-clopen set of $\mathrm{X}$ including $\mathrm{w}$ which is $\mathrm{Sb}-\mathrm{T}_{2}$-ordered $\mathrm{sp}$.

Proof. If $(\mathrm{X}, \mathcal{T}, \mathcal{R})$ is $\mathrm{Sb}-\mathrm{T}_{2}$-ordered $\mathrm{sp}$, then $\mathrm{X}$ is the required $\mathcal{J}$-(resp. $\mathcal{D}$-) b-clopen set of $\mathrm{X}$ for each $\mathrm{w} \in \mathrm{X}$.

Conversely, $(\mathrm{w}, \mathrm{z}) \notin \mathcal{R}$. From the hypothesis, there is an $\mathcal{J}$-b-clopen set $\mathcal{S}$ in X such that w $\in \mathcal{S}$. If $\mathrm{z} \in \mathcal{S}$, then there is nothing to prove. If $\mathrm{z} \notin \mathcal{S}$, Then, $\mathrm{z} \in \mathcal{S}^{\mathrm{c}}$ is $\mathcal{D}$-b-clopen set of $\mathrm{X}$ containing $\mathrm{z}$. Thus, $(\mathrm{X}, \mathcal{T}, \mathcal{R})$ is $\mathrm{Sb}-\mathrm{T}_{2}$-ordered sp. Similarly, we can prove the theory of $\mathcal{D}$-b-clopen of $\mathrm{X}$.

Theorem 4.7. A Top-o.sp $(\mathrm{X}, \mathcal{T}, \mathcal{R})$ is $\mathrm{Sb}$ - $\mathrm{T}_{2}$-ordered sp if and only if for every couple of points $(\mathrm{w}, \mathrm{z}) \notin \mathcal{R}$ in $\mathrm{X}$, there is a mapping $\mathrm{F}$ of $\mathrm{X}$ into $\mathrm{Sb}-\mathrm{T}_{2}$-ordered $\mathrm{sp}\left(\mathrm{Y}, \mathcal{T}^{*}, \mathcal{R}^{*}\right)$ such that

(i) $\quad(\mathrm{F}(\mathrm{w}), \mathrm{F}(\mathrm{z})) \notin \mathcal{R}^{*}\left(\right.$ resp. $\left.(\mathrm{F}(\mathrm{z}), \mathrm{F}(\mathrm{w})) \notin \mathcal{R}^{*}\right)$

(ii) $\quad F$ is increasing (resp. decreasing)

(iii) $\quad \mathrm{F}$ is $b$-irresolute.

Proof. Let $(X, \mathcal{T}, \mathcal{R})$ is $S b-T_{2}$-ordered sp. The identity mapping is then a necessary mapping.

Conversely, let $(w, z) \notin \mathcal{R}$. From the premise, there is an increasing $b$-irresolute mapping $\mathrm{F}$ from $X$ into a $S b$ - $T_{2}$-ordered sp with $(\mathrm{F}(w), \mathrm{F}(z)) \notin \mathcal{R}{ }^{*}$. Consequently, there are disjoint $b$-open sets $\mathcal{S}^{*}$ and $\mathcal{K}^{*}$ in $Y$ containing $\mathrm{F}(w)$ and $\mathrm{F}(z)$, respectively, where $\mathcal{S}^{*}$ is $\mathcal{J}$ - and $\mathcal{K}^{*} \mathcal{D}$-set. Since $\mathrm{F}$ is an increasing $b$-irresolute mapping, therefore $\mathrm{F}^{-1}\left(\mathcal{S}^{*}\right)$ is an $\mathcal{J}$-b-open set, $w \in \mathrm{F}^{-1}\left(\mathcal{S}^{*}\right)$, and $\mathrm{F}^{-1}\left(\mathcal{K}^{*}\right)$ is $\mathcal{D}$ - $b$-open set containing $z$. Also, $\mathrm{F}^{-1}\left(\mathcal{S}^{*}\right) \cap \mathrm{F}^{-1}\left(\mathcal{K}^{*}\right)=\emptyset$. Thus, $X$ is $S b-T_{2}$-ordered sp. Likewise, we can verify the theorem for the decreasing mapping.

Corollary 4.8. Let $\mathrm{Y}$ be an $\mathrm{Sb}-\mathrm{T}_{2}$-ordered sp. If $\mathrm{F}$ is order-preserving, then it is b-irresolute mapping from $(\mathrm{X}, \mathcal{T}, \mathcal{R})$ into $\left(\mathrm{Y}, \mathcal{T}^{*}, \mathcal{R}^{*}\right)$. Then, $\mathrm{X}$ is $\mathrm{Sb}$ - $\mathrm{T}_{2}$-ordered sp.

Proof. The proof can be obtained from the hypothesis and Theorem 4.7.

Theorem 4.9. The product family of $\mathrm{Sb}-\mathrm{T}_{2}$-ordered sp's is also $\mathrm{Sb}-\mathrm{T}_{2}$-ordered $\mathrm{sp}$.

Proof. Let $\left\{\left(\mathrm{X}_{\alpha}, \mathcal{T}_{\alpha}, \mathcal{R}_{\alpha}\right): \alpha \in \mathrm{J}\right\}$ be a family of $\mathrm{Sb}$ - $\mathrm{T}_{2}$-ordered sp and $(\mathrm{X}, \mathcal{T}, \mathcal{R})$ be the product order space. If $\left(\mathrm{w}_{\alpha}\right),\left(\mathrm{z}_{\alpha}\right) \in \mathrm{X}$ such that $\left(\left(\mathrm{w}_{\alpha}\right),\left(\mathrm{z}_{\alpha}\right)\right) \notin \mathcal{R}$, then there exists $\beta \in \mathrm{J}$ such that $\left(\mathrm{w}_{\beta}, \mathrm{z}_{\beta}\right) \notin \mathcal{R}_{\beta}$. Thus, there are disjoint b-open sets $\mathcal{S}_{\beta}, \mathcal{K}_{\beta}$ in $\mathrm{X}_{\beta}$ containing $\mathrm{w}_{\beta}$ and $\mathrm{z}_{\beta}$, respectively, where $\mathcal{S}_{\beta}$ is $\mathcal{J}$-set and $\mathcal{K}_{\beta}$ is $\mathcal{D}$-set. We define $\mathcal{S}=\prod_{\alpha \in \mathrm{J}} \mathcal{S}_{\alpha}$ such that $\mathcal{S}_{\beta}=\mathrm{X}_{\beta}$ if $\alpha \neq \beta$ and $\mathcal{K}=\prod_{\alpha \in \mathrm{J}} \mathcal{K}_{\alpha}$, such that $\mathcal{K}_{\beta}=\mathrm{X}_{\beta}$ if $\alpha \neq \beta$. Then, $\mathcal{S}$ is $\mathcal{J}$-b-open set containing $\left(\mathrm{w}_{\alpha}\right)$ and $\mathcal{K}$ is $\mathcal{D}$-b-open set containing $\left(\mathrm{z}_{\alpha}\right)$. $\mathcal{S}$ and $\mathcal{K}$ are disjoints. Hence, $(\mathrm{X}, \mathcal{T}, \mathcal{R})$ is $\mathrm{Sb}$ - $\mathrm{T}_{2}$-ordered sp.

Theorem 4.10. If $(\mathrm{X}, \mathcal{T}, \mathcal{R})$ is $\mathrm{Sb}-\mathrm{T}_{2}$-ordered $\mathrm{sp}$, then the graph of the order of $\mathrm{X}$ is a b-closed subset of $\mathrm{X} \times \mathrm{X}$.

Proof. Let $\mathrm{Q}$ be the graph of the order of $\mathrm{X}$ and $(\mathrm{w}, \mathrm{z}) \in \mathrm{X} \times \mathrm{X}-\mathrm{Q}$. Then, $(\mathrm{w}, \mathrm{z}) \notin \mathcal{R}$. By the hypothesis, there exist disjoint $\mathcal{J}$-b-open set $\mathcal{S}$ and $\mathcal{D}$-b-open set $\mathcal{K}$ with $\mathrm{w} \in \mathcal{S}, \mathrm{z} \in \mathcal{K}$. Therefore, $\mathcal{S} \times \mathcal{K}$ is a b-open subset of $\mathrm{X} \times \mathrm{X}$ containing $(\mathrm{w}, \mathrm{z})$. Also, $\mathcal{S} \times \mathcal{K} \cap \mathrm{Q}=\emptyset$. Thus, $(\mathrm{w}, \mathrm{z}) \in \mathcal{S} \times \mathcal{K} \subseteq$ $\mathrm{X} \times \mathrm{X}-\mathrm{Q}$. Hence, $\mathrm{Q}$ is b-closed.

Remark 4.11. $\mathrm{Sb}-\mathrm{T}_{2}$-ordered $\mathrm{sp}$ is not a hereditary property in general, as we show in the following example. 
Example 4.12. Let $X=\{x, y, z\}, \tau=\{X, \emptyset,\{x\},\{z\},\{x, z\}\}$, and $\mathcal{R}$ be the partial order relation on $\mathrm{X}$, defined as $\mathcal{R}=\triangle \mathrm{U}\{(y, x)(y, \mathrm{z})\}$. Then, $(\mathrm{X}, \mathcal{T}, \mathcal{R})$ is Sb-T ${ }_{2}$-ordered sp. Let $\mathcal{A}=\{x, y\} \subseteq \mathrm{X}$. Then we have $\mathcal{T}_{\mathcal{A}}=\{\mathcal{A}, \emptyset,\{x\}\}$ and $\mathcal{R}_{\mathcal{A}}=(\mathcal{A} \times \mathcal{A}) \cap \mathcal{R}=\{(x, x),(y, x),(y, y)\}$. Clearly, $(x, y) \notin \mathcal{R}_{\mathcal{A}}$. Then, there exists an $\mathcal{J}$-b-open set $\{x\}$ containing $x$, the only $\mathcal{D}$-b-open set containing $y$ is $\mathcal{A}$, and clearly, $\{x\} \cap \mathcal{A} \neq \emptyset$. Consequently, $\left(\mathcal{A}, \mathcal{T}_{\mathcal{A}}, \mathcal{R}_{\mathcal{A}}\right)$ is not Sb-T2-ordered subsp of $(\mathrm{X}, \mathcal{T}, \mathcal{R})$.

Theorem 4.13. Every open subspace of $\mathrm{Sb}-\mathrm{T}_{2}$-ordered $\mathrm{sp}$ is $\mathrm{Sb}-\mathrm{T}_{2}$-ordered $\mathrm{sp}$.

Proof. Let $(\mathrm{X}, \mathcal{T}, \mathcal{R})$ be an $\mathrm{Sb}-\mathrm{T}_{2}$-ordered sp and $\mathcal{A} \subseteq \mathrm{X}$ be an open subset of X. Let $(\mathrm{w}, \mathrm{z}) \notin \mathcal{R}$ in $\mathcal{A}$, then $(\mathrm{w}, \mathrm{z}) \notin \mathcal{R}$ in $\mathrm{X}$. By the hypothesis, there are disjoint $\mathcal{J}$-b-open set $\mathcal{S}$ and $\mathcal{D}$-b-open set $\mathcal{K}$ in $\mathrm{X}$ such that $\mathrm{w} \in \mathcal{S}, \mathrm{z} \in \mathcal{K}$. Since $\mathcal{A}$ is an open set, so by Proposition 2.7 part (2), $\mathcal{S} \cap \mathcal{A}$ and $\mathcal{K} \cap \mathcal{A}$ are disjoint b-open sets in $\mathcal{A}$ containing w and z, respectively, $\mathcal{S} \cap \mathcal{A}$ is $\mathcal{J}$-set in $\mathcal{A}$, and $\mathcal{K} \cap \mathcal{A}$ is $\mathcal{D}$-set in $\mathcal{A}$. Thus, $\mathcal{A}$ is $\mathrm{Sb}-\mathrm{T}_{2}$-ordered subsp.

Theorem 4.14. Let $\mathrm{F}:(X, \mathcal{T}, \mathcal{R}) \rightarrow\left(Y, \mathcal{T}^{*}, \mathcal{R}^{*}\right)$ be a bijective and $b$-closed mapping and $(X, \mathcal{T}, \mathcal{R})$ be a $S b$ - $T_{2}$-ordered sp. Then, $\left(Y, \mathcal{T}^{*}, \mathcal{R}^{*}\right)$ is also $S b$ - $T_{2}$-ordered sp where $\mathcal{R}^{*}$ is the quotient order brought by $\mathrm{F}$.

Proof. Let $p \in Y$ and $Q=\cap\{\mathcal{B}: \mathcal{B}$ is $\mathcal{J}$-b-closed set $\mathcal{J}$ - $b$-nbhd of $p\}$. Then, $[p,-] \subseteq Q$. Let $p^{*} \in$ $[p,-]$. Then, $\left(p, p^{*}\right) \notin \mathcal{R}^{*}$. Since $\mathrm{F}$ is onto and $\mathcal{R}^{*}$ is the quotient, there exist $w, z \in X$ such that $\mathrm{F}(w)=p, \mathrm{~F}(z)=p^{*}$ and $(w, z) \notin \mathcal{R}$, implies $z \notin[w,-]$, and since $X$ is $S b$ - $T_{2}$-ordered sp. Hence, there exists $\mathcal{A}$ an $\mathcal{J}$-b-closed $\mathcal{J}$-b-nbhd of $w$ such that $z \notin \mathcal{A}$. Since $\mathrm{F}$ is injective and b-closed, then $\mathrm{F}(\mathcal{A})$ is an $\mathcal{J}$-b-closed $\mathcal{J}$-b-nbhd of $\mathrm{F}(\mathrm{w})=\mathrm{p}$ and $\mathrm{F}(\mathrm{z})=\mathrm{p}^{*} \notin \mathrm{F}(\mathcal{A})$, implies $\mathrm{p}^{*} \notin \mathrm{Q}$. Hence, $[\mathrm{p},-]=$ $\mathrm{Q}$ and, consequently, $\left(\mathrm{Y}, \mathcal{T}^{*}, \mathcal{R}^{*}\right)$ is $\mathrm{Sb}-\mathrm{T}_{2}$-ordered sp. The proof in the case of $\mathrm{p}$ is similar.

Remark 4.15. From Propositions 2.3 and 3.4, we obtain the following diagram which shows the implications between the separation axioms that we have come across in this paper and the examples that there is no other implication that holds between them.

$$
\begin{aligned}
& \mathrm{S} \text { - } \mathrm{T}_{2} \text {-ordered } \mathrm{sp} \Rightarrow \mathrm{Sb}-\mathrm{T}_{2} \text {-ordered } \mathrm{sp} \Rightarrow \mathrm{b}-\mathrm{T}_{2} \text {-sp } \\
& \Downarrow \quad \Downarrow \quad \Downarrow \\
& \mathrm{S} \text { - } \mathrm{T}_{1} \text {-ordered } \mathrm{sp} \Rightarrow \mathrm{Sb} \text { - } \mathrm{T}_{1} \text {-ordered } \mathrm{sp} \Rightarrow \mathrm{b} \text { - } \mathrm{T}_{1} \text {-sp } \\
& \Downarrow \quad \Downarrow \quad \Downarrow \\
& \mathrm{S} \text { - } \mathrm{T}_{0} \text {-ordered } \mathrm{sp} \Rightarrow \mathrm{Sb} \text { - } \mathrm{T}_{0} \text {-ordered } \mathrm{sp} \Rightarrow \mathrm{b} \text { - } \mathrm{T}_{0} \text {-sp }
\end{aligned}
$$

\section{References}

1. Nachbin, L. 1965. Topology and ordered. D. Van Nostrand Inc.Princeton, New Jersey.

2. McCartan, S. D. 1968. Separation axioms for topological ordered spaces. Mathematical Proceedings of the Cambridge Philosophical Society, 64: 965-973.

3. Arya, S. D. and Gupta, K. 1991. New separation axioms in topological ordered spaces. Indian J. pure appl. Math., 22:461-468.

4. Leela, D. S. and Balasubramanian, G. 2002. New separation axioms in ordered topological spaces. Indian J. pure appl. Math., 33(7):1011-1016.

5. Shanthi, S. and Rajesh, N. 2018. Separation axioms in topological ordered spaces. Italian Journal of Pure and Applied, 40:464-473.

6. Künzi, H. A. and Richmond, T. A. 2004. Completely regularly ordered spaces versus $T_{2}$-ordered spaces which are completely regular. Topology and its Applications, 135:185-196.

7. Dhanapakyam, C. and Indirani, K. 2019. On $\beta \mathrm{g}^{*}$ Closed Sets in Topological Ordered Spaces International Journal of Mathematics Trends and Technology, 65(1):7-8.

8. Amarendra babu, V. and Aswini, J. 2017. g*-Closed sets in topological ordered spaces. International Journal of Advanced in Management, Technology and Engineering Sciences, 7(12):113-125.

9. Ashaea, G. S. and Yakub, Y. Y. 2019. Filter bases and $j-\omega$-perfect mappings. Ibn al-Haitham Journal for Pure and Applied Science, 32(2):165-177.

10. Ali, H. J. and Abbas, H. F. 2018. On $\theta$-totally disconnected and $\theta$-light mappings. Ibn al-Haitham Journal for Pure and Applied Science, 31(2):179-185.

11. Humadi, N. K. and Ali, H. J. 2020. New types of perfectly supra continuous functions. Iraqi Journal of Science, 61(4):811-819. DOI: 10.24996/ijs.2020.61.4.13.

12. Andrijevic, D. 1996. On b-open set. Mat. Vesink, 48:59-64. 
13. Ekici, E. and Caldas, M. 2004. Slightly $\gamma$-continuous functions. Bol. Soc. Parana. Mat., (3) 22(2):63-74.

14. Caldas, M. and Jafari, S. 2007. On some applications of b-open sets in topological spaces. Kochi J. Math., 2:11-19.

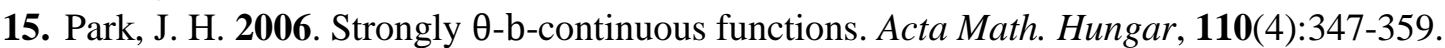

16. Abo Elhamayle Abo Elwafa, M. A. 2009. on bitopological ordered spaces. Master Thesis, Mansoura University, Mansoura, Egypt.

17. Noiri, T., Mashhour, A. S., Hasancin, I. A., and El-Deeb, S. N. 1982. A note on S-closed subspaces. Math. Sem. Notes Kobe Univ., 10:431-435. 